\title{
A SCALABLE MULTICAST PROTOCOL WITH QOS GUARANTIES
}

\author{
Abderrahim Benslimane and Omar Moussaoui ${ }^{2}$ \\ Laboratoire d'Informatique(LIA) - Université d'Avignon
}

BP 1228 - 84911 AVIGNON CEDEX 9

Email: benslimane@lia.univ-avignon.fr

Abstract: The technological advances in communication networks and computers are largely contributed to the developments of multimedia applications such as the videoconference. These applications require an efficient management of the Quality of Service (QoS) and consist of a great number of participants. In order to support such applications, we propose a hierarchical communication architecture and multicast protocols allowing scalability. This architecture allows to guarantee QoS requirements. In fact, it allows to reduce efficiently the connection number and the communication delays. We use simulation to analyze and compare the performances of the proposed multicast protocols to the direct architecture. Simulation results confirm our purposes that are the reduction of the network charge and the average delay.

Key words: Multicast, Quality of Service, Hierarchical architecture, Shortest path, Corebased tree, Source-based tree.

\section{INTRODUCTION}

The emergence of multimedia applications, like the videoconference, raised several constraints on the subjacent networks. Indeed, these applications require the transport of heterogeneous data such as text, voice, and video. Thus, it is difficult to transmit over packet networks the produced streams because of the generated rate

\footnotetext{
${ }^{1}$ This work is supported by CNRS/JemSTIC grant N ${ }^{\circ}$ SUB/2002/004/DR16. ${ }^{2}$ Currently at LICP, Université de Cergy-Pontoise.
}

The original version of this chapter was revised: The copyright line was incorrect. This has been corrected. The Erratum to this chapter is available at DOI: 10.1007/978-0-387-35703-4_21 
(especially for the video) and their strong temporal constraints. It is then necessary to be able to offer better quality of service: guaranties of bandwidth, mimum delay and jitter.

Multimedia applications were strongly deployed inside companies on private networks. However, a network such as the Internet does not support them yet. Internet does not support any QoS: it is based on the best effort model. Many works are in progress to allow QoS guarantee or at least service differentiation. The paradigm connectionless (datagram) offers a great adaptability for data transfer but is badly adapted to the new multimedia services. The end-to-end communications (or unicast) are not adapted any more to the multipartite applications.

Several multicast protocols were proposed in the literature. However, the majority of them are not scalable. Indeed, these protocols are badly adapted to the networks of great dimension like the Internet. So, techniques based on hierarchical trees represent a very promising solutions. Nevertheless, studies are primarily related to only theoretical aspects without taking account implementation. In the same way, several work was proposed to support QoS in networks $[1,4,8,11,13]$.

In this work, we propose a hierarchical architecture allowing efficient communication between group multicast members and supporting guaranties of QoS. The architecture consists on grouping members, according to their localization and to their response to the QoS requirements of the application, into disjoint groups. We use two different methods to connect these groups. The first one consists on using the concept of core based tree. Whereas work in [7] use rendezvous points in static way our protocol determinates rendezvous points in a dynamic way. The second one consists on using shortest path tree to connect groups between them. In the two methods, we take into account criteria of QoS.

The rest of the paper is organized as follow. In section 2, we present an overview of QoS and multicast protocols. In section 3, we propose a hierarchical architecture for efficient multicast. The construction of this architecture is done in two steps. In the first step, we construct local subgroups. Each subgroup elects a process as its server. In the second step, we connect elected servers with hierarchical tree by using two different methods. In section 4 , we evaluate the performances of the hierarchical architecture by simulations of different examples with NS tool.

\section{QOS AND MULTICAST OVERVIEW}

With the introduction of multimedia applications requiring guaranties of QoS, the multicast problem becomes more challenging. Routing multicast protocols can be classified in two categories: core-based and source-based multicast protocols 10, 12].

A specific tree or source-based is built starting from a given source, so that it is necessary to build several trees for the same multicast group if this group includes several sources. It is unidirectional tree from a source to the receivers while using the notion of shortest path tree (SPT). Each branch of the tree is the shortest path from the sender to each group member. Source-based routing is currently used in Distance Vector Multicast Routing Protocol (DVMRP) [14], Dense Mode protocol- 
Independent Multicasting (PIM-Dense) [6], and Multicast Open Shortest Path First (MOSPF) [9].

On the other hand, shared tree or center-based protocol is established once to connect all multicast group members to the center or core nodes that constitutes the root of the spanning tree. It is bidirectional because there is no distinction between sources and receivers. The core based tree (CBT) [2] and PIM Sparce Mode (PIMSM) [7] are well known examples of shared tree protocol. Each sender transmits messages to the multicast group toward the core. Then, the messages are distributed to group members along the path from the core.

The shortest path (in number of hops) is usually the shortest delay path. So, the receivers in the multicast tree obtain a QoS satisfaction. However, since each individual receiver must have a shortest path from the source, the scalability problem occurs. The network nodes must store a number of states bounded by $\theta(G . S)$ where $G$ is the number of multicast groups in the network and $S$ represents the average number of sources per multicast group.

The Core-based multicast protocols are highly suitable for sparse groups and scalable for large networks. They provide excellent bandwidth conservation for receivers. The number of state to be stored does not depend on the number of sources, so that it is bounded by $\theta(G)$.

In addition to the need of scalability, group based multimedia applications also demand stringent QoS requirements such as the minimum end-to-end delay, jitter delay and the efficient use of bandwidth.

Several single-path routing protocols such as delay-constrained unicast routing (DCUR) [11] and residual delay maximizing (RDM) [13] have been proposed for QoS unicast routing that can be used, as well, for multicast routing. These protocols typically use delay and cost tables in making routing decisions during QoS connection establishment. In contrast to these unicast protocols, many multicast routing protocols have been proposed in order to increase QoS guaranties by finding the best path among multiple candidate paths that satisfy the QoS.

In the Spanning Join protocol (SJP) [4], the new member broadcasts join-request messages in its neighborhood to find on-tree nodes. Whenever an on-tree node receives the message, it sends a reply message back to the new member. The new member may receives multiple reply messages corresponding to multiple candidate paths. Each reply message collects the QoS properties of the path it traverses. The best path is then, chosen.

In the QoSMIC [8], the search for candidate paths consists of two procedures, local search and tree search which can be executed in parallel or sequentially. The local search is equivalent to spanning join, except that only a small neighborhood is searched. The tree search handles the case when there is no on-tree node in the neighborhood checked by the local search. In the tree search, the new member contacts a designated manager node that is responsible for ordering a subset or ontree nodes to establish a path from them to the member. The new member chooses the best path among these candidate paths.

In QRMP [5], two sequential procedures are introduced: single-path mode and multiple-path mode according to network conditions. The protocol starts and continues with single-path mode until it reaches a node that has insufficient resources to satisfy the join request. When such a node is encountered, the protocol changes the mode. 
All these above protocols do not take into account all the requirements of good multicast routing protocol such as scalability, end-to-end delay, bandwidth. For example, SJP and QoSMIC are not scalable to Internet because of high message overhead due to their flooding nature. QMRP has the same problem in multipath mode. It also incurs a high joining time due to its sequential invocation of multipath mode from single-path mode.

To overcome these limitations, we propose a hierarchical architecture allowing efficient communication between group multicast members and supporting guaranties of QoS. The architecture consists on grouping members, according to their localization and to their response to the QoS requirements of the application, into disjoint groups. We use two different methods to connect these groups. The first one consists on using the concept of core based tree. Whereas work in [7] use rendezvous points in static way our protocol determinates rendezvous points in a dynamic way. The second one consists on using shortest path tree to connect groups between them. Thus, we propose scalable multicast protocols, based on short path first, for networks of great dimension and sensitive to QoS.

\section{HIERARCHICAL ARCHITECTURE FOR MULTICAST GROUPE}

Hierarchical trees offer properties particularly interesting to support multicast in wide-area networks. It is not question to use Steiner trees that are too complexes and requiring information on the location of the multicast group members. For that, we were interested in the core-based tree and shared tree protocols to overcome the scalability problem.

\subsection{System environment}

We consider a multicast group $M$ constituted of $N$ processes $\left\{P_{1}, \ldots, P_{n}\right\}$ distributed geographically on inter-connected different networks. These processes have different identities and communicate between them toward the Internet network. Moreover, they participate at the same multimedia application such as videoconferencing. Communication between two processes $\mathrm{P}_{\mathrm{i}}$ and $\mathrm{P}_{\mathrm{j}}$ can take different path.

\subsection{Local groups construction}

Considering the high number of participants in multicast group M, it appears essential to divide them into local groups according to the concentration in the various areas of the Internet to ensure guaranties of QoS. In other term, this decomposition allows to use efficiently the bandwidth, to reduce the consumption of resources, to optimize the delay and to ensure communications between processes. The construction of local groups was introduced in [1]. The proposed algorithms suppose that processes communicate between them via virtual channels. In this work, we use real paths, i.e. physical links existing between the hosts and routers 
participating in the multicast group. To build these local groups, we propose the construction of neighboring sets, transit groups and local groups.

\subsubsection{Neighboring set}

In this first step, we construct for each process $P_{i}$ a neighboring set $V_{i}$ by using conditions on the delay and Time to Live TTL of messages. Given delay threshold D and a live range of messages TTL (hop number). A process $P_{j}$ belongs to $V_{i}$ if TTL from $P_{i}$ to $P_{j}$ (decremented hop by hop) is not null and the round-trip delay between $P_{i}$ and $P_{j}$ is less than D. The path from $P_{i}$ to $P_{j}$ is the shortest one.

\subsubsection{Transit group}

To guarantee QoS effectively, each process builds its own transit group by carrying out evaluations, initially given in [1], on the capabilities of processing and buffering of media units.

- Test on processing capabilities- This test allows checking if the process has sufficient processing capabilities to handle data flows coming from its neighbors. A process $P_{i}$ must be able to process all media units generated in the same time by its neighbors in a time duration not exceeding the time allocated to the processing of media units.

- Test on available buffering size - It consists to check if process $P$ is able to store all media units coming on different paths between $\stackrel{i}{P}_{i}$ and its neighboring processes to compensate variation delays (jitter) of these paths.

By these two tests, each process $P_{i}$ constructs its one transit group $g t r_{i}$ from its neighboring set $V_{i}$. For example, we have in figure 1.a: $V_{1}=\{2,4,5,9\}, V_{2}=\{1,4,5$, $8,9\}$ and $V_{3}=\{4,6,7\}$.

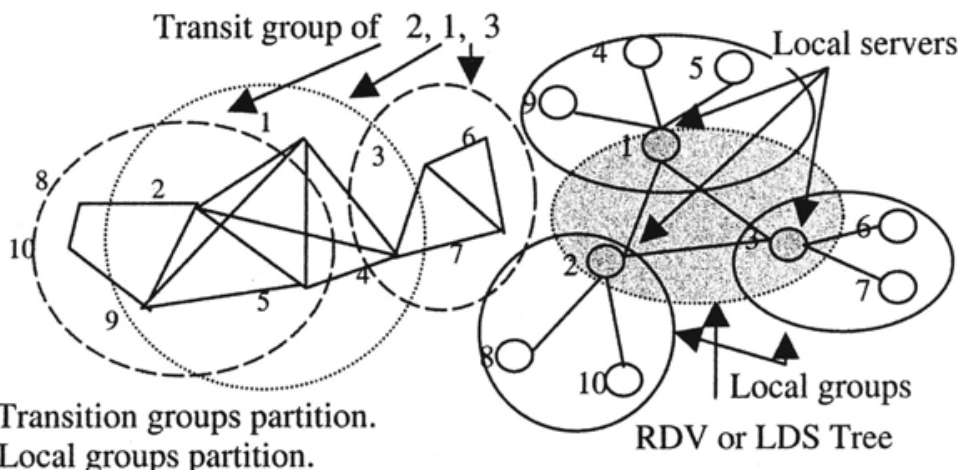

(a) Transition groups partition.

(b) Local groups partition.

Figure 1. Construction of local groups 


\subsubsection{Grouping and election}

Once transit groups are built, each process knows the members of its own set and the paths which connect it to them. However, some processes can belong to several transit groups at the same time (Figure 1.a). To solve this problem, a mechanism must take place to remove useless connections. Each process must broadcasts its transit group to its neighbors. Once these messages are received, each receiver select the maximum of the processes existing simultaneously in these groups of transit (construction of maximum cover set). If a process belongs to several local groups, it will be put in that having the smallest size. This allows to balance the number of the processes in these groups.

Thus, the multicast group $\mathrm{M}$ is divided into local sub-groups. Each element of $\mathrm{M}$ belongs to only one local group (Figure 1.b). Then a local server (process having some characteristics: reducing the average or round-trip delay, or having the maximum processing capacity, or opening to the outside, or the smallest identity) and a secondary server are elected to represent each local group. In other term, a process communicates with the other participants of the multicast group only through the server of its local group. The principal role of the secondary server is to replace the local server if this last wishes to leave multicast group or it crashes (break down).

\subsection{Hierarchical tree construction between servers}

Now, let us consider that $\mathrm{K}$ local groups are built and each one has its local server. Let $\Gamma=\left\{S_{1}, . ., S_{K}\right\}$ the set of these local servers which can be distributed in different networks. Remark that the number of these servers can be very high. Moreover, in a videoconference several sources can belong to the same multicast group. Consequently, it is necessary to build a multicast tree which allows to link these servers while reducing the participants of the multicast group. So, we propose the use of two methods: core-based tree and SPT tree. The first one is called RDV (RenDezVous) hierarchical architecture and the second LDS (Link Direct Shortest path) hierarchical architecture. Complete description and algorithms of these two methods can be found in [2].

\subsubsection{RDV Hierarchical Architecture}

Core-based tree allows to build shared tree in a simple way and to minimize used resources. Moreover, the performances (cost and delay average) are reasonable in the case of many-to-many applications (several sources and several receivers). Nevertheless, the choice of the rendez-vous points, with their associated problems, make difficult their implementation in networks on a large scale. In the literature and in a general, only one rendezvous point is used and it is selected in static ways (fixed initially by the manager). In this work, we determine the rendezvous points in dynamic way. Moreover, we propose the use of several rendezvous points to avoid the congestion problem of the traffic when only one rendezvous point is used. 


\section{Algorithm description:}

We suppose that $S_{p} \in \Gamma$ is the local server of the group where is located the first source which want to broadcast media units to the other members of the multicast group $M$. This server represents the first rendezvous point $R P_{i}$. Given a threshold delay $D^{\prime}$ that is selected by taking account of the network extent.

At the beginning, $\mathrm{RP}_{1}$ does not have any information on the addresses of the other servers. Consequently, it broadcasts hop by hop an initialization message $\operatorname{INIT}\left(R P_{1}\right.$, $a d r G, T T L$ ) including the address of the rendezvous point $R P_{i}$, a time to live TTL and group multicast address adrG. All members of $M$ that are not servers delete the received message. However, each local server $S_{i}$ sends an acknowledge message $A C K\left(S_{i}, R P_{1}\right)$ to $R P_{1}$. When $R P_{1}$ receives acknowledgement message from $S_{i}$, it computes the round-trip delay $r d_{1}$. If this delay is smaller than the threshold $D^{\prime}$, it selects the server $S_{i}$ and puts it in its autonomous domain $D A_{1}$ (a set of processes). Then, it stores in $\bar{S}$ all other servers which are not in its domain $D A_{1}: \bar{S}=\Gamma-D A_{1}$. After construction of $D A_{1}, R P_{1}$ memorizes the computed delay between it and each server $S_{\mathrm{i}}$ of $\bar{S}$ in a matrix $M A T$, allowing to store the round-trip delays between the elements of $\bar{S}$ and the existing rendezvous points in the multicast tree, in order to determinate the next rendezvous point (Table 1). Then, it sends the message $\operatorname{SUCCESSOR}\left(S_{i}, R P_{1}, \bar{S}, M A T\right)$ to the nearest server $S_{i}$, which does not belong to its domain $D A_{1}$. In other words, that which is linked to $R P_{1}$ by a path having the smallest value of round-trip delay $r d_{1 i}$, strictly higher than $\mathrm{D}^{\prime}$, and which belongs to $\bar{S}$.

Table 1. Round-trip delays between rendezvous points and servers of $S$

\begin{tabular}{|c|c|c|c|c|}
\hline $\mathrm{RP} \backslash S$ & $\mathrm{~S}_{\mathrm{i}}$ & $\mathrm{S}_{\mathrm{j}}$ & $\mathrm{S}_{\mathrm{k}}$ & $\mathrm{S}_{1}$ \\
\hline $\mathrm{RP}_{1}$ & $\mathrm{rd}_{1 \mathrm{i}}$ & $\mathrm{rd}_{1 \mathrm{j}}$ & $\mathrm{rd}_{1 \mathrm{k}}$ & $\mathrm{rd}_{11}$ \\
\hline $\mathrm{RP}_{2}$ & $\mathrm{rd}_{2 \mathrm{i}}$ & $\mathrm{rd}_{2 \mathrm{i}}$ & $\mathrm{rd}_{2 \mathrm{i}}$ & $\mathrm{rd}_{2 \mathrm{i}}$ \\
\hline$\ldots$ & & & & \\
\hline
\end{tabular}

When a local server $S_{i}$ receives message $\operatorname{SUCCESSOR}\left(S_{i}, R P_{p}, \bar{S}, M A T\right)$, it understands that it constitutes a new rendezvous point, we noted $R P_{2}$. The new rendezvous point $R P_{2}$ play the role of initiating server of the set $\bar{S}$ : the set of servers which are not in the domain of $R P_{1}$. Thanks to message SUCCESSOR, $R P_{2}$ knows addresses of all processes in $\bar{S}$, then it sends to them message $\operatorname{JOIN}\left(R P_{2}, S\right)$ in unicast way. When server $S_{\mathrm{j}}$ receives this message, it replies with $A C K\left(S_{\mathrm{j}}, R P{ }_{2}\right)$ to $R P_{2}$. Then, $R P_{2}$ computes the minimum round-trip delays between it and elements of $\bar{S}$ and carries out the following operations: 
- It select all servers of $\bar{S}$ having a round-trip delay smaller or equal to the threshold D' and puts them in its autonomous domain $\mathrm{DA}_{2}$.

- It removes from $\bar{S}$ the selected servers: $\bar{S}=\bar{S}-D A 2$.

- It removes the columns of the matrix MAT corresponding to the elements of its domain $D A_{2}$.

- It adds a line in MAT to store the minimum round-trip delays between it and all servers of $\bar{S}$ : those which do not belong to any domain.

- It searches the smallest value of delays in MAT and takes the couple $(R P$, $S_{q}$ ) corresponding to this value. Thus, we determined dynamically the next rendezvous point $\mathrm{S}_{\mathrm{q}}$, among the servers of $\bar{S}$, which father is $\mathrm{RP}_{\mathrm{p}}$ that is a rendezvous point already created. In other term, $\mathrm{S}_{q}$ is a server of the set $\bar{S}$ and it is the nearest to the existing rendezvous point $R P_{p}$ while taking the round-trip delay as metric.

- It sends message $\operatorname{SUCCESSOR}\left(S_{q}, R P_{p}, \bar{S}, M A T\right)$ to the new rendezvous point $S_{9}$, noted $R P_{3}$.

- Finally, $\mathrm{RP}_{2}$ joins members of its domain $\mathrm{DA}_{2}$ and its rendezvous point father $\mathrm{RP}_{1}$ toward the SPT tree which root is $\mathrm{RP}_{2}{ }_{2}$.

After the new rendezvous point $\mathrm{RP}_{3}$ receives the message $\operatorname{SUCCESSOR}\left(S_{q}, R P_{p}\right.$, $\bar{S}, M A T)$, it makes the same thing as $\mathrm{RP}_{2}$ and so on until emptiness of $\bar{S}$. In other words, until all servers are connected to the multicast tree.

\subsubsection{LDS Hierarchical Architecture}

In this method, we are based on SPT tree to build links between servers. SPT trees are used for their simplicity and their excellent performances for multimedia flows or conversational flows (low delay and low concentration). Nevertheless, this type of tree consumes many resources (many states, number of links used). To overcome this problem we construct SPT tree on demand. In other words, we built SPT tree for a local server $S_{j}$, if and only if the local group of this server contains at least one source wanting to multicast multimedia flows to the other memberships multicast group.

\section{Algorithm description:}

Same manner as in the first method, we suppose that $S_{j}$ is the local server of the group where is located the source which want to multicast, the first, media units. $S_{j}$ broadcasts hop by hop an initialization message $\operatorname{INIT}\left(S_{\mathrm{j}}\right.$, adrG, TTL). As this message contains the address of multicast group, only members of this group have the right to deliver the message. At the reception of this message, all members of $\mathrm{M}$ which are not servers delete it. On the other hand, each local server joins the server $S_{\mathrm{j}}$ by the shortest path by using unicast routing protocol. Once SPT tree is built, the root server $S_{\mathrm{j}}$ sends a message $\operatorname{INFO}\left(S_{\mathrm{j}}, \Gamma\right)$, including the addresses of all local servers of multicast group $M$, to its successor servers. After reception of this message, each successor server takes note of the others. For the moment, we built 
only one multicast tree constituted by SPT tree linking the local servers and the other SPT trees of local groups. Then, if a participant $m_{\mathrm{i}}$, of multicast group M, wants to become source of multimedia flow, it must request it to its server $S_{k}$ by sending message REQUEST $\left(m_{\mathrm{i}}, S_{\mathrm{k}}\right)$. Then, $S_{\mathrm{k}}$ joins all other servers with the shortest path by using an unicast routing protocol. Then, it multicasts the flow coming from $\mathrm{m}_{\mathrm{i}}$ to the other elements of its own local group and to the other local servers by using the built multicast tree.

\subsection{Management of the hierarchical architecture}

Once the hierarchical architecture is built, a mechanism must take place to manage the add and remove of multicast group members. We describe such mechanism in the following:

- If a simple process wants to leave multicast group $M$, then it is removed from its local group by all other processes of the same group via their local server.

- If a new process $P_{n}$ wants to join multicast group $\mathrm{M}$, it broadcasts the message $\operatorname{JOIN}\left(a d r P_{n}{ }^{n}, a d r G, T T L\right)$ to $P_{n}$, where $a d r P_{n}$ is the address $P_{n}$, $a d r G$ is the address of multicast group $\mathrm{M}$ and $T T L$ is the maximum time to live of the message. Thanks to this message, $P_{n}$ requests to the servers of its region for a participation to the session. At the reception of $\operatorname{JOIN}(\operatorname{adr} P$, $a d r G, T T L)$ with TTL greater to zero, all simple participants delete it. However, each local server carries out evaluations on its processing capabilities and its available memory. All servers receiving this message and which are able to accommodate $P_{n}$, replies with a message $\operatorname{RECP}\left(a d r S_{i}\right.$, $B d_{i}$, size) where $a d r S_{i}$ is the address of a sender server $S_{i}, B d_{i}$ is the space memory of $S_{i}$ available and size is the number of elements of its local group $G l_{i}$ In the case where $P_{n}$ receives several RECP, it chooses the local group of the server having the smallest size or having the maximum space memory available. Once $P_{n}$ has chosen the local group, it joins its server by the shortest path.

- If a local server $S_{i}$ wants to leave multicast group M (or it crashes), it requests the secondary server of its local group to replace it. It informs the other members of its group about the new server. Also, it informs the secondary server about the members of their local group and the other local servers which are linked to it. This allows the new server to manage the members of its local group and to join the other local servers as follows:

- In case of RDV architecture, it joins the RP of its autonomous domain by the shortest path.

- In case of LDS architecture, it joins each server of a local group in which belongs at last one source by the shortest path.

- If a new server wants to join the multicast group M, it broadcasts a message at the multicast address adrG. When receiving this message, each local server replies by sending a message including its address and indication if its local group contains or not a multicast source. In case of the RDV 
architecture, this message includes also the address of the RP of the autonomous domain of the sender server. The new server join the multicast group as follows:

O In case of RDV, it joins the nearest RP by the shortest path.

- In case of LDS, it joins each server of a local group in which belongs at last one source by the shortest path.

\section{SIMULATIONS AND ANALYSIS}

In these simulations, we are interested with the scalability problem that arise when multimedia applications in multicast groups are to be executed. The way with which the communication is done between participants influences the guarantee of $\mathrm{QoS}$ and the scalability. So, for these raisons we focus the analysis on the communication between local groups. For this purpose, we implement RDV and LDS architectures and compare them to the DIR, DIRect architecture connecting members directly by the shortest paths, by using NS2 simulator.

With a first program (in $\mathrm{C}++$ language), we create a connected graph constituted of 2000 nodes which are set in a matrix of 5000x5000 and linked between them in a random way. We choose 8 multicast sources of multimedia stream. With a second program, implementing Ford's algorithm, we create 50 sub-groups. As a metrics, we use the shortest path with a given TTL $=5$ hops to consider the delay. Also, this program allows to designate, randomly, the groups where sources are located. Moreover, for each group a node with a smallest identity is chosen as a local server. For RDV, we have selected 5 local servers as rendezvous points RP. Then all other local servers are connected to these RP. For LDS, we connected directly, each local server of the group where is located one source to all others. For DIR, we used the shortest paths between each source and the other nodes.

Simulation parameters are: simulation time is $100(\mathrm{~s})$, generation rate of each source is 200 (media units/s) and media unit size is 500 bytes. The scheduling of multicast for each source is as follows: source 1 from $0 \mathrm{~s}$ to $80 \mathrm{~s}$, source 2 from $10 \mathrm{~s}$ to 80 s, source 3 from 20 s to 80 s, source 4 from 30 s to 80 s, source 5 from 50 s to $80 \mathrm{~s}$, source 6 from 60 s to 80 s, source 7 from 70 s to 80 s, source 8 from 70 s to 100 s.

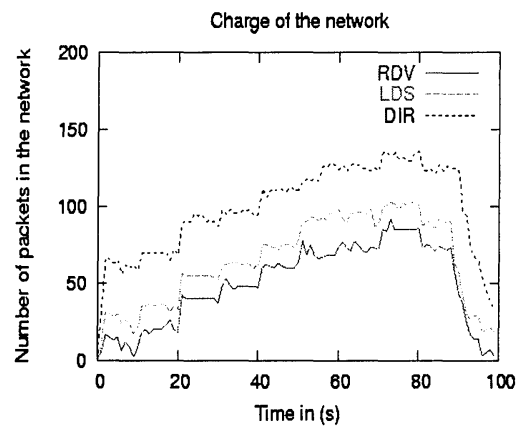

a. Charge of the network.

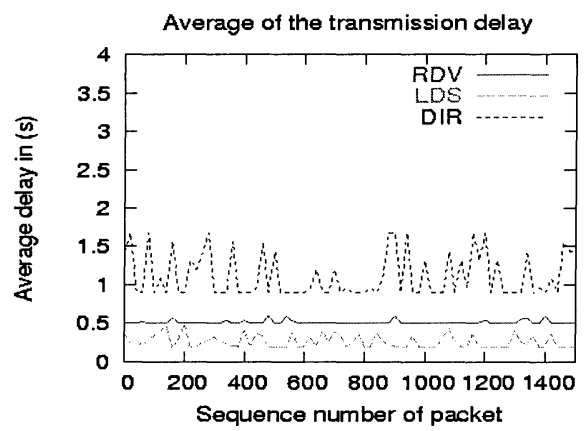

b. Average of the transmission delay.

Figure 2. Network of 2000 nodes 
At each $0.1 \mathrm{~s}$, we count the number of packets in the network. This number depends on the number of multicast sources. In figure 2.a, we observe that the number of packets is very high between $70(\mathrm{~s})$ and 80 (s) because the 8 sources, broadcast streams at the same time. Also, we compute the average of transfer delay for each packet, from its emission by a source until its arrival to all destinations (Figure 2.b). The average of transfer delay of a packet is the time necessary so that this packet is delivered by all receivers of the multicast group.

To see the influence of the size of the multicast group, we increase the number of nodes in the network to 3000 and the number of sources to 10 . For this simulation, we create 66 sub-groups and take $6 \mathrm{RP}$ for RDV.

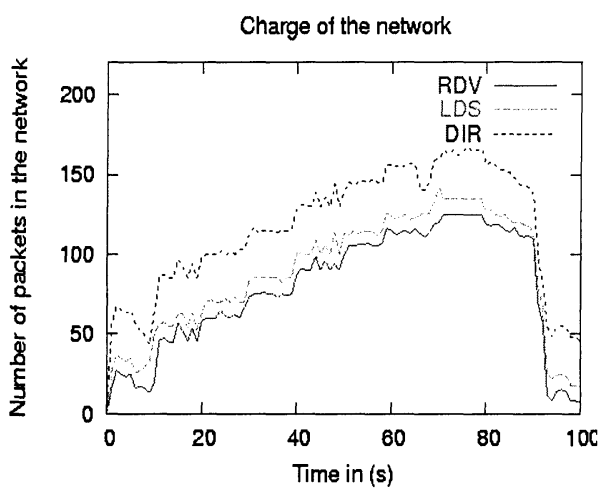

a. Charge of the network.
Average of the transmission delay

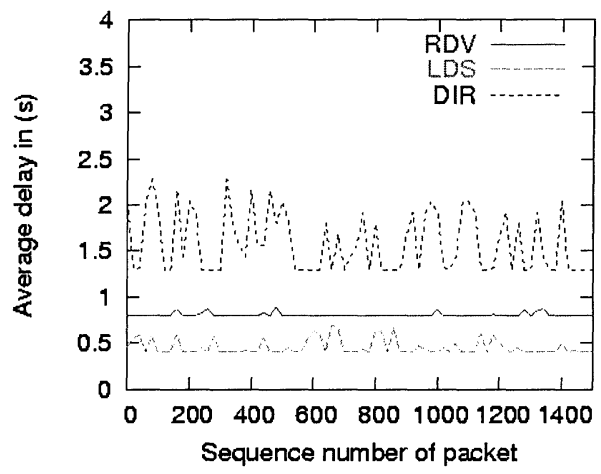

b. Average of the transmission delay.

Figure 3. Network of 3000 nodes

Simulation results in figures 3.a and 3.b show the same conclusions as in the first simulation. Moreover, we remark the increase of bandwidth rate and the average of the transfer delay proportional with the number of the participants in multicast group. In figures 2.b and 3.b, we remark that the average of transfer delay in RDV architecture remains about constant for most packets. This is because in RDV, packets sent by the sources take the same ways to arrive to the receivers.

In parallel to the above simulations, we have extracted data corresponding to the end-to-end delays. We considered packets sent from one specific source to some receivers. We computed the amount of time spent between the emission and the reception of each packet. Then, we divided this time by the number of packets.

Curves represented in figures 4.a and 4.b show that the end-to-end delay is better in DIR compared to LDS and RDV. Indeed, in DIR the sources are connected directly by the shortest paths to the destinations. On the other hand, in RDV and LDS packets sent by the sources must obligatorily pass by the local servers to reach theirs destinations.

Also, we remark that the end-to-end delay is smaller in LDS than in RDV. This is because in LDS the servers of the local groups containing sources are directly connected to the other local servers. However, in RDV the local servers are connected between them via RP. 


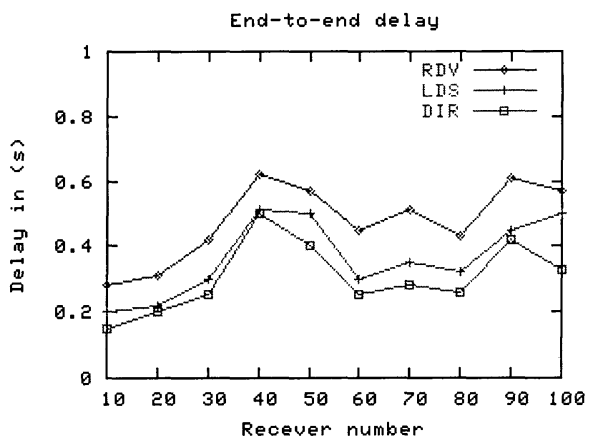

a- Network of 2000 nodes.

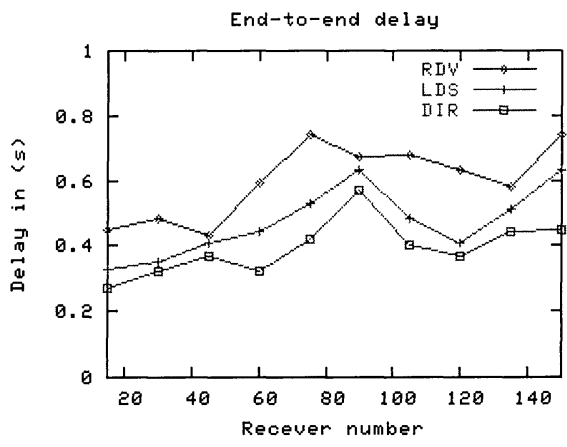

b. Network of 3000 nodes.

Figure 4. End-to-end delay

\section{CONCLUSION}

Within the framework of multimedia applications, we proposed a hierarchical architecture of communication and scalable multicast protocols. This architecture allows to guarantee QoS. Indeed, it allows to effectively reduce the occupation rate of bandwidth, the transmission delays and the memory size necessary to store the media units devoted to the multimedia synchronization. Moreover it allows a dynamic and effective management of participants to take into account failures.

To implement this architecture, we proposed the use of two methods: core-based tree and SPT tree. The first one is called RDV (RenDezVous) hierarchical architecture and it uses only one multicast tree by using rendezvous points in a dynamic way. It allows to optimize the used resources and to reduce the load factor of the bandwidth. The second one, LDS (Link Direct Shortest path) hierarchical architecture uses very significant resources in the case of several sources and several receivers. Nevertheless, it is more powerful for the multimedia applications. Indeed, it allows to minimize the communication delays considerably and to effectively reduce the concentration of traffic compared to the first method. It would be preferable to use the 1rst method for many-to-many applications not requiring strong temporal constraints.

We carried out simulations of the proposed architectures on different examples by using NS2 simulator. The results obtained show the reducing of the used bandwidth and the average of transmission delays.

\section{REFERENCES}

1. A. Benslimane et A. Abouaissa, "Dynamical Grouping Model for Distrubuted Real Time Causal Ordering", Computer Communications journal, Vol. 25, pp. 288-302, January 2002. 
2. A. Benslimane, "Multicast Protocols: Scalability and QoS Studies", Technical Recherche Repport, April 2003.

3. A. Ballardie, "Core Based Trees (CBT version 2) Multicast routing; protocol specification", RFC 2189, September 1997.

4. K. Carlberg and J. Crowcroft, "Building Shared Trees Using a one-to-many Joining Mechanism", Computer Communication Review, January 1997, pp. 5-11.

5. S. Chen, K. Nahrstedt, and Y. Shavitt, "A QoS-Aware Multicast Routing Protocol“, IEEE INFOCOM, May 2000.

6. S.Deering et al., "Protocol Independent Multicast-Dense Mode (PIM-DM): Protocol Specification", Internet draft, June 1999.

7. D. Estrin et al., "Protocol Independent Multicast-Sparse Mode (PIM-SM): Protocol Specification", RFC 2362, June 1998.

8. M. Faloutsos, A. Banerjea and R. Pankaj, "QoSMIC: Quality of Service sensitive Multicast Internet protocol”, SIGCOMM'98, September 1998.

9. J. Moy, "Multicast Routing Extensions for OSPF", Commun. ACM, Vol. 37, pp. 61-66, August 1994.

10.B. Mukherjee L.H. Sahsrabuddhe, "Multicast Routing Algorithms and Protocols", IEEE Network, January/February 2000.

11. H.F. Salama, D.S. Reeves and Y. Viniotis, "A Distributed Algorithm for DelayConstrained Unicast Routing”, IEEE Infocom'97.

12.A. Striegel and G. Manimaran, "A Survey of QoS Multicasting Issues", IEEE Communications Magazine, June 2002, pp. 82-87.

13. R. Sriram, et al., "Preferred Link-Based Delay-Constrained Least Cost Routing in Wide Area Networks", Computer Communication, Vol. 21, N 18, 1998.

14.D. Waitzman and C. Partridge, "Distance Vector Multicast Routing Protocol", RFC 1075, November 1988. 